\title{
Preface
}

\section{Hip Pain in the Athlete: The Source is Key}

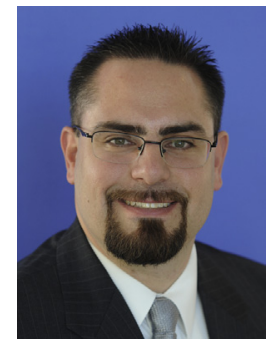

Dustin L. Richter, MD

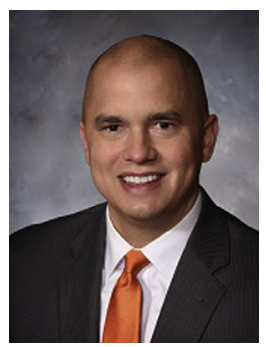

F. Winston Gwathmey, MD Editors

Compared with the diagnosis and management of pain in the knee and shoulder, diagnosis and management of hip pain in the adolescent and young adult population had been relatively neglected until the twenty-first century. During the past 2 decades, there has been an explosion in the understanding of hip pathologic condition and management of hip conditions in the younger population without arthritis. Improved indications and modern techniques have been developed that make the field of hip arthroscopy relatively safe with a low complication rate.

Examining a patient with hip pain can seem quite daunting, because of more than 20 muscles that cross the hip joint and because of both intraarticular and extraarticular sources of pain. Thankfully, we've compiled a group of experts in the field to shed some light on how to work up and treat these patients to achieve optimal outcomes. We would like to thank our friends and colleagues who have given their time and talent to contribute to this issue of Clinics in Sports Medicine. They are leaders in their field, many of whom serve as team physicians with a vested interest in returning athletes to their prior level of competition. All our contributors are dedicated to advancing the understanding and management of hip pain in the athlete through surgeon, physician, trainer, and patient education. In this issue of Clinics in Sports Medicine, you will learn to properly evaluate and image patients with pain in the hip region. The diagnosis and management of both intraarticular and extraarticular hip issues are reviewed. Additional excellent articles on special topics, including the adolescent athlete and sex-based differences in injury rates, help round out the current issue.

Enjoy perusing articles to learn about what radiographs and advanced imaging are necessary to make an accurate diagnosis? If a patient has hip dysplasia: Who can safely be treated with an arthroscopic approach and who may require an acetabular osteotomy? What are the indications for doing a labral reconstruction versus a labral repair? How do you manage stress fractures in elite athletes or pelvic avulsion injuries 
xvi Preface

in the adolescent athlete? These are just some of the topics we hope to provide guidance on by sharing our knowledge through practice and evidence-based medicine.

Dustin L. Richter, MD

Department of Orthopaedic Surgery University of New Mexico Health Sciences Center MSC 105600

Albuquerque, NM 87131, USA

F. Winston Gwathmey, MD Department of Orthopaedic Surgery University of Virginia School of Medicine

PO Box 800159

Charlottesville, VA 22908, USA

E-mail addresses:

Dustin.richter1818@gmail.com (D.L. Richter) FWG7D@hscmail.mcc.virginia.edu (F.W. Gwathmey) 